\title{
Cloning and Expression of Two Soluble Acid Invertase Gene Isoforms from Rhododendron
}

\author{
He Lisi, Su Jiale', Liu Xiaoqing, Li Chang, and Chen Shangping \\ Institute of Horticulture, Jiangsu Academy of Agricultural Sciences, 50 Zhongling Street, Nanjing \\ 210014, China
}

\begin{abstract}
AdDitional INDEX words. azalea, bioinformatics analysis, enzyme activity, Ericaceae, floral development, soluble sugar, transcriptional level

Abstract. Soluble acid invertase [SAI (Enzyme Commission 3.2.1.26)] plays an important role in catalyzing the hydrolysis of sucrose into hexoses and regulates floral development. Full-length cDNAs encoding $R h S A I I$ and $R h S A I 2$ isoforms were cloned from Rhododendron hybrid 'Yuqilin' and they exhibited high amino acid sequence identity $(89 \%)$ to each other. The protein sequences contain highly conserved motifs present in all SAIs, including the $\beta$-fructosidase motif N-D-P-(D/N), a putative active site W-E-C-(I/V)-D, and R-D-P. The expression of $R h S A I 1$ and $R h S A I 2$ genes was under spatial and temporal control. Expression of both $R h S A I 1$ and $R h S A I 2$ genes was most abundant in stems, and expression was lowest in roots and leaves, respectively. The expression of $R h S A I 2$ was significantly lower than that of $R h S A I 1$ in all organs. During floral development, $R h S A I 1$ was highly expressed at the earliest stage (Stage I), decreased until Stage III, and increased again at the terminal stage. The pattern of $R h S A I 2$ expression was distinctly different, showing a continuous increase during floral development. Consistent with the levels of $\boldsymbol{R} \boldsymbol{h S A I 1}$ expression, SAI activity decreased during floral development and was inversely correlated with the soluble sugar content. Abundant expression of RhSAII at the transcriptional level in addition to high SAI activity during the initial stages of floral development may play a vital role in supplying the energy needed for rapid cell division and growth of flowers.
\end{abstract}

Sucrose is the principal sugar in plants and plays a vital role in plant growth and development (Rolland et al., 2006) by serving as a critical molecule for carbohydrate and energy flow from source organs to sink organs (Salerno and Curatti, 2003). The distribution of sucrose between the flower and the rest of the shoot system may influence floral development as a result of the gradient in sugars between the source organs (leaves and stems) and sink organs (Laia and Sergi, 2012; van Doorn, 2004). Developing flowers act as a sink and the rate of carbohydrate flow from source to sink is related to a number of enzyme activities of which invertases provide a crucial contribution (Sood et al., 2006). Invertases, which catalyze the irreversible hydrolysis of sucrose into glucose and fructose, are a group of ubiquitous enzymes that can be divided into acid, neutral, or alkaline groups according to their solubility, $\mathrm{pH}$ optima, and subcellular localizations. Acid invertase exists in the apoplast (insoluble) and the vacuole (can be called soluble acid invertase or vacuole invertase), whereas neutral or alkaline invertase is localized to the cytoplasm (Roitsch and Gonzalez, 2004; Tao et al., 2010). The activities of invertases play key roles in carbohydrate metabolism and in the modulation of plant development (Anne et al., 2011; Jain et al., 2008; Koch, 1996). For example, increased activity of acid invertase might be correlated with the senescence of asparagus (Asparagus officinalis) stored in air (Hurst et al., 1997). Two broccoli (Brassica oleracea var. italica) acid invertase cDNAs were cloned and RNA blot analysis showed that their transcripts accumulate during senescence of broccoli florets (Coupe et al., 2003). Meanwhile, there is more research conducted on the expression of SAI genes

Received for publication 30 Aug. 2013. Accepted for publication 29 Nov. 2013. This research was supported by the Jiangsu Provincial Agriculture Science and Technology Innovation Fund [grant No. cx(11)4052] and the Jiangsu Provincial Science and Technology Supporting Program (grant No. BE2011325).

${ }^{1}$ Corresponding author. E-mail: suj166@aliyun.com. in relation to fruit development. In grape (Vitis vinifera) berries, two SAI genes termed GIN1 and GIN2 are cloned and found to be abundantly expressed before fruit veraison, and their expression is attenuated during grape maturation (Davies and Robinson, 1996). Levels of PSS-AIVI and PSS-AIVI transcripts, which are isogenes of SAIs in japanese pear (Pyrus pyrifolia) fruit, reach their maximum at $34 \mathrm{~d}$ after full bloom and decrease rapidly during fruit maturation (Yamada et al., 2007).

The genus Rhododendron, belonging to the family Ericaceae, comprises almost 1000 species with a worldwide distribution but which is concentrated within the sub-alpine and alpine zones of Nepal, India, Malaysia, and China (Singh et al., 2009). Species and cultivars within the genus Rhododendron are often used as ornamentals as a result of their beautiful and colorful flowers. Rhododendron hybrid 'Yuqilin' has doubled pink flowers with stamen petalody and is one of the most popular azalea cultivars in China because of its vigorous growth and hardiness. However, no studies have reported on the physiological mechanism of sucrose metabolism and related gene expression in Rhododendron 'Yuqilin'. To gain insight into the connection between SAI gene transcription and sugar content during flower development, we cloned two isoforms of a SAI gene from Rhododendron 'Yuqilin' and investigated their function in flower development by analyzing the expression of their transcripts, enzyme activity, and soluble sugar content.

\section{Materials and Methods}

Materials. Plants were grown in a greenhouse at the Horticultural Institute in Jiangsu Province, China. Rhododendron hybrid 'Yuqilin' was grown in a defined potting mix (3 sphagnum:1 perlite). Four-year-old plants with uniform growth and apparent flower buds were chosen as the experimental material to examine expression patterns of the SAI gene. The entire floral 
Table 1. Primers used for two soluble acid invertase gene isoforms isolation in Rhododendron.

\begin{tabular}{lll}
\hline Primer & \multicolumn{1}{c}{ Oligonucleotide sequence $\left(5^{\prime}-3^{\prime}\right)$} & \multicolumn{1}{c}{ Application } \\
\hline Forward primer $_{1}$ & TACCATCTATTCTACCAGTACAA & PCR of $R h S A I 1$ partial cDNA sequence \\
Reverse primer & AAAAAATCAGGACACTCCCACAT & PCR of $R h S A I 1$ partial cDNA sequence \\
Forward primer $_{2}$ & TATAACCCGGAGAACGACAAGTG & PCR of $R h S A I 2$ partial cDNA sequence \\
Reverse primer $_{2}$ & ACTGTTCTTCCTCCTTGGCCAAA & PCR of $R h S A I 2$ partial cDNA sequence \\
Outer primer $_{1}$ & TTGAACGGTGTTTGGACTGG & first of $R h S A I 13^{\prime}$ RACE \\
Inner primer $_{1}$ & TCGCTATCGGGTCAAAGGTG & second of $R h S A I 13^{\prime}$ RACE \\
Outer primer $_{2}$ & TATAACCCGGAGAACGACAAGTG & first of $R h S A I 23^{\prime}$ RACE \\
Inner primer $_{2}$ & TAACGCCTGTCTATTTCTAC & second of $R h S A I 23^{\prime}$ RACE \\
Primer $_{1}$ & TTGCTGAATCGGGGTTGTACTGGTAGAATA & $5^{\prime}$ RACE of RhSAII (Clontech, Tokyo, Japan) \\
Primer $_{2}$ & TAATCCACCCGCAACCCGATACCCACG & $5^{\prime}$ RACE of RhSAI2 (Clontech)
\end{tabular}

$\mathrm{PCR}=$ polymerase chain reaction; $\mathrm{RACE}=$ rapid amplification of $\mathrm{cDNA}$ ends.

Table 2. Gene-specific primers sequence for detection by real-time quantitative polymerase chain reaction.

\begin{tabular}{lll}
\hline Gene & \multicolumn{1}{c}{ Forward primer $\left(5^{\prime}-3^{\prime}\right)$} & \multicolumn{1}{c}{ Reverse primer $\left(5^{\prime}-3^{\prime}\right)$} \\
\hline Actin & GCAGTGTTCCCCAGTATT & TCTTTTCCATGTCATCCC \\
RhSAII & ACGCCCAAGAAGGCACAT & CCCATAGTCCATCCGCAAC \\
RhSAI2 & TTCCGAGGACGGTAGTGTT & CCACCAATAACCGCATAGAG \\
\hline
\end{tabular}

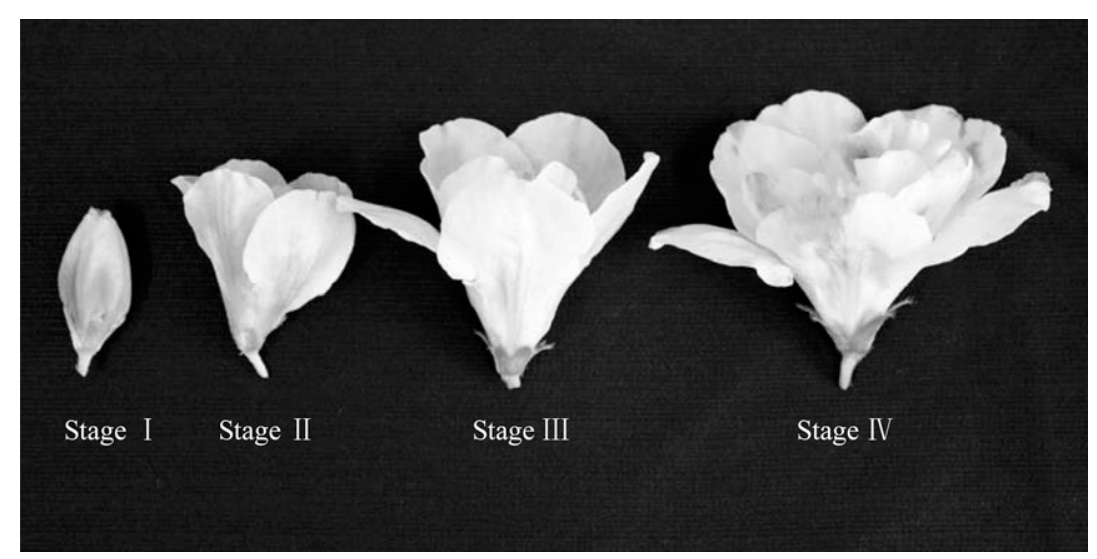

Fig. 1. Detail of floral developmental stages in Rhododendron. The entire floral development period was divided into four different stages till end flowering for a period of 20 to $25 \mathrm{~d}$, including Stage I (closed bud, dark pink petals), Stage II (first blossoming, pink petals), Stage III (full-blooming, light pink at the central of the petals and gradually change to white at the edge), Stage IV (end flowering, petals color change into white with a little bit light pink at central).

development period was divided into four different stages until end flowering for a period of 20 to $25 \mathrm{~d}$ (Fig. 1), including 3 to $4 \mathrm{~d}$ from Stage I (closed bud, dark pink petals) to Stage II (first blossoming, pink petals), 2 to $3 \mathrm{~d}$ from Stage II to Stage III (full-blooming, light pink at the central of the petals and gradually change to white at the edge), and 15 to $18 \mathrm{~d}$ from Stage III to Stage IV (end flowering, petals color change into white with a little bit light pink at central).

RNA EXTRACTION AND SYNTHESIS OF CDNA. The vegetative tissues, including leaves, stems, and roots at Stage III, were sampled along with flower petals at four stages of floral development and flash frozen in liquid nitrogen and then stored at $-70{ }^{\circ} \mathrm{C}$ for further use. Total RNA was isolated using the method described by Meng et al. (2006). From this total RNA, cDNA was synthesized by using a PrimeScript ${ }^{\circledR}$ RT reagent Kit with gDNA Eraser (TaKaRa, Tokyo, Japan). This cDNA served as a template for polymerase chain reaction (PCR). The primers were designed based on conserved sequences within SAI genes derived from Prunus persica (GenBank accession number JQ412748), Agave tequilana (JN790063), Nicotiana tabacum (AJ305044), and Ipomoea batatas (AY037938) (Table 1). Using reverse transcription-PCR, two partial cDNA sequences representing $R h S A I 1$ and $R h S A I 2$ isoforms were cloned from Rhododendron 'Yuqilin'. Based on the sequence of the resulting partial cDNAs, we used the $3^{\prime}$ and $5^{\prime}$ rapid amplification of cDNA ends (RACE) method to isolate fulllength cDNAs of Rhododendron SAI genes. For $3^{\prime}$-RACE, cDNA derived from leaves at Stage I was used in two rounds of PCR with outer primer and inner primer used to amplify. Following sequencing of the resulting PCR products, the sequences of the $5^{\prime}$ ends of RhSAI1 and RhSAI2 genes were obtained by using a SMARTer ${ }^{\mathrm{TM}}$ RACE cDNA Amplification Kit (Clontech, Tokyo, Japan) according to the manufacturer's instructions with primer $_{1}$ and primer $_{2}$, respectively.

Cloning AND SeQuencing. PCR products were separated by $1 \%$ agarose (Sigma, St. Louis, MO) gel electrophoresis, and bands were purified using the Agarose Gel DNA Purification Kit (Version 2.0; TaKaRa). The extracted products were ligated into the pMD18-T cloning vector (TaKaRa) and transformed into competent DH5a Escherichia coli cells (Trans, Beijing, China). Integrity of the recombinant plasmids was first examined with BamHI and HindIII restriction enzymes (TaKaRa) and confirmed by sequencing at the Shanghai Sangon Biological Engineering Technology \& Services Co. (Shanghai, China).

Bioinformatics ANALYSIS. Sequence and multiple alignment analyses were performed using DNAMAN 5.0 (Lynnon Corp., Vaudreuil, Quebec, Canada). Sequences that exhibited high amino acid identity (more than 70\%) to the SAI proteins from Rhododendron were selected to build a phylogenetic tree. The phylogenetic tree was constructed in MEGA 5.05 (Tamura et al., 2011) using the neighbor-joining method.

GENE EXPRESSION ANALYSIS. The real-time quantitative PCR (Q-PCR) was performed on a CFX96 ${ }^{\mathrm{TM}}$ Real-Time System (C1000 Thermal Cycler; Bio-Rad, Hercules, CA). Expression of an actin isoform (JN105299) was monitored as an internal control. The designed primers used in gene expression analysis 
are shown in Table 2. Q-PCR reactions were performed using SYBR $^{\circledR}$ Premix Ex Taq ${ }^{\mathrm{TM}}$ (Perfect Real Time; TaKaRa) and contained $12.5 \mu \mathrm{L}$ of $2 \times$ reaction mix, $0.5 \mu \mathrm{L}$ of $50 \times$ ROX Reference Dye II (TaKaRa), $2 \mu \mathrm{L}$ of cDNA solution as a

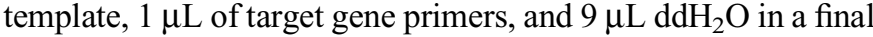
volume of $25 \mu \mathrm{L}$. The thermal profile was as follows: $50^{\circ} \mathrm{C}$ for $2 \mathrm{~min}$; $95^{\circ} \mathrm{C}$ for $5 \mathrm{~min}$; and 40 cycles of $95^{\circ} \mathrm{C}$ for $15 \mathrm{~s}, 51^{\circ} \mathrm{C}$ for $15 \mathrm{~s}$, and $72{ }^{\circ} \mathrm{C}$ for $40 \mathrm{~s}$. Data were automatically gathered using CFX Manager software (V1.6.541.1028; Bio-Rad). Relative gene expression measurements were calculated using the $2^{-\triangle \triangle \mathrm{Ct}}$ comparative threshold cycle $(\mathrm{Ct})$ method (Schmittgen and Livak, 2008). The expression of $R h S A I I$ transcript in flower petals at Stage I of floral development was used as the comparator sample (1.00) for relative gene expression analysis in Q-PCR analysis of gene expressions in different tissues at Stage III and in petals at four stages of floral development.

Quantification OF SOLUble sugars. Soluble sugars (sucrose, glucose, and fructose) were extracted by high-performance liquid chromatography (HPLC) as described (Munneé-Bosch and Lalueza, 2007) with slight modifications. Flower petal samples $[0.5 \mathrm{~g}$ fresh weight $(\mathrm{FW})]$ from Stage I to Stage IV were ground in liquid nitrogen and extracted with $80 \%(\mathrm{v} / \mathrm{v})$ ethanol (Dingguo
Biotechnology Co., Beijing, China) for $60 \mathrm{~min}$ at room temperature. After centrifugation, supernatants were dried completely under rotary evaporators (4003; Heidolph Instruments, Schwabach, Germany) and reconstituted in $1 \mathrm{~mL}$ of water. The extracts were immediately passed through a $0.45-\mu \mathrm{m}$ Millipore filter (Sartorius, Berlin, Germany). Soluble carbohydrates were isocratically separated using an Agilent 1100 HPLC system (Agilent, Palo Alto, CA) with a CARBOSep CHO-620 CA column $(6 \times 250 \mathrm{~mm}, 10 \mu \mathrm{m}$; Trangenomic, Omaha, NE) using double-distilled water as a solvent at a flow rate of $1 \mathrm{~mL} \cdot \mathrm{min}^{-1}$. Injection volume was $15 \mu \mathrm{L}$ and detection was carried out with a differential refractometer (SCH2000; SCH, Rostock, Germany). Authentic standard substances obtained from Sigma were used for quantification.

EXTRACTION AND ASSAY OF SAI ACTIVITY. For the extraction of SAI, flower petal samples $(0.5 \mathrm{~g} \mathrm{FW})$ from Stage I to Stage IV were homogenized at $4{ }^{\circ} \mathrm{C}$ in Tris- $\mathrm{HCl}(0.1 \mathrm{M}, \mathrm{pH}$ 6.8; Sigma) containing $2 \mathrm{~mm}$ phenylmethylsulfonyl fluoride (Sigma), $1 \mathrm{~mm}$ ethylenediaminetetraacetate (Sigma), $10 \mathrm{~mm}$ 2-mercaptoethanol (Sigma), $10 \mathrm{~mm} \mathrm{Na}$ ascorbate (Sigma), and 10\% (w/v) Polyclar VT (Sigma). The homogenate was centrifuged at $10,000 g_{n}$ for $20 \mathrm{~min}$ at $4{ }^{\circ} \mathrm{C}$ and the supernatants were used for enzyme assays.

\begin{tabular}{|c|c|}
\hline & AACCAAAATAAAAATGAACTCCCATTCTTCCCCTCCTGAAGACCTGGAAAGATCGGCCGCO \\
\hline & $\begin{array}{lllll}D & \mathrm{~L} & \mathrm{E} & \mathrm{R} & \mathrm{S}\end{array}$ \\
\hline 9 & CTCGGCTGCCGGACCACCCTCCCTCTCCGGCGGATCACCGCCGACCCTTCAAGGGTCTCGTCGGAATATTTGTCTO \\
\hline 8 & $\begin{array}{llllllllllllllllllllll}D & H & P & P & S & P & A & D & H & R & R & P & F & K & G & L & V & G & I & F & V & S\end{array}$ \\
\hline 57 & CTCTTAATGTCGICTTTGGTTGCTTTAATCCTCAATCAAGACCCTCGTCCCCGGTCTAACTTCAATGACGACCZ \\
\hline 4 & $\begin{array}{llllllllllllllllllllllllll}M & L & L & M & S & S & L & V & A & L & I & L & N & Q & D & P & R & P & R & S & N & F & N & D & D & Q\end{array}$ \\
\hline 5 & GGGAGAGTACATCTCCTTCAATGCCGGTGCCGGATAGTTTAATGCCGCCGTCGAGGGGGGTGGCGCAGGGGGTGTO \\
\hline 0 & 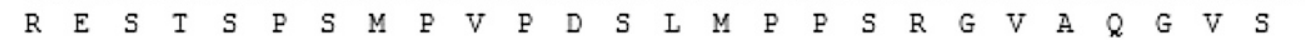 \\
\hline 13 & AGAAGGCGGTCCGGGAATTCTCCGGTAGCGGCCCGGTTITCCCTTGGACTAATGCTATGCTGGCTTGGCAGAGGACTT \\
\hline 6 & 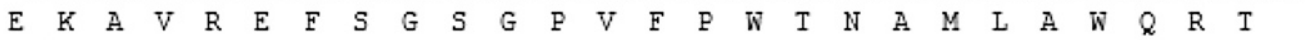 \\
\hline & TACCATTITCAGCCGGAAAAAAACTGGATGAATGATCCTGATGGICCATTGCATCACATGGGATGGTACCACCTAT \\
\hline 2 & 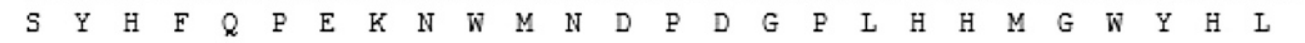 \\
\hline 69 & TCTACCAGTACAACCCCGATTCAGCAATTTGGGGCAACATAACATGGGGCCACGCAGTATCAAGGGACCTGATCCACT \\
\hline 48 & 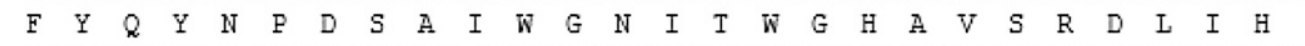 \\
\hline 7 & GGCTCTACCTCCCCATCGCCATGGTCCCCGATCACTGGITCGATTTGAACGGTGTTTGGACTGGGTCTGCTACCCTCC \\
\hline 74 & 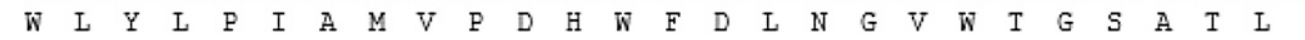 \\
\hline 25 & TCCCCGACGGTCAGATCATCATGTTGTACACCGGTGATACCGATAACGCCGTGCAGGTGCAAAACCTAGCGTATCCCG \\
\hline 0 & 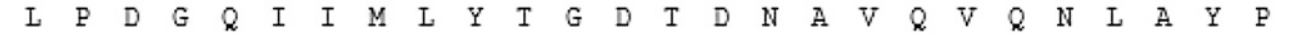 \\
\hline 03 & CCAACTTATCTGATCCCCTCCTCCTAGATTGGGTCAAGTATGAACAAAACCCGGTAATTGTTCCCCCACCCGGAATTG \\
\hline 26 & 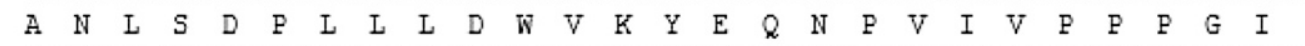 \\
\hline$E 1$ & GACTAACGTATTTTCGGGACCCGAGTACAGCATGGTACGCCCAAGAAGGCACATGGCGGGTCGCTATCGGGTCAAAGG \\
\hline 52 & 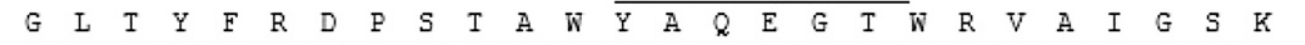 \\
\hline 59 & TGAATAAAACGGGTACTGCCCTTGIGTACCAAACCACTAATTTTACTAGCTTCGAGCTTATGGATGGGGTAATGCATG \\
\hline 78 & 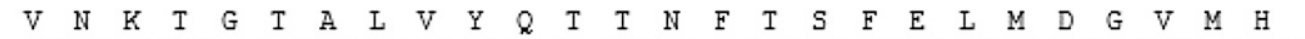 \\
\hline 37 & CGGTTCCGGGTACAGGTATGTGGGAGTGCATAGATTTTTACCCGGTCTCAACAAACAGTACAGTTGGGTTGAACTCGT \\
\hline 04 & 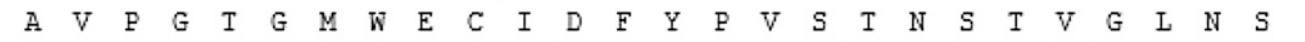 \\
\hline 015 & CGGITATTGGGCCGGATGTCAAGCACGTGCTCAAGGCAAGTTIGGATGATGATAAGAAGGATITTTATGCACTCGGGA \\
\hline 30 & 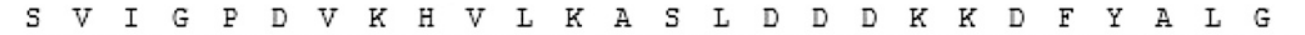 \\
\hline 993 & CATACGATCTAAGTAATAACACATGGACACCGGATGACCCGGAGATAGATGTGGGTATCGGGTTGCGGATGGACTATG \\
\hline 56 & $\begin{array}{llllllllllllllllllllllllllll} & \mathrm{Y} & \mathrm{D} & \mathrm{L} & \mathrm{S} & \mathrm{N} & \mathrm{N} & \mathrm{T} & \mathrm{W} & \mathrm{T} & \mathrm{P} & \mathrm{D} & \mathrm{D} & \mathrm{P} & \mathrm{E} & \mathrm{I} & \mathrm{D} & \mathrm{V} & \mathrm{G} & \mathrm{I} & \mathrm{G} & \mathrm{I} & \mathrm{R} & \mathrm{M} & \mathrm{D} & \mathrm{Y}\end{array}$ \\
\hline .71 & GGAATTTTACGCATCAAAAACATTCTATGACCCGACAAAGCAGAGGAGGATCTT \\
\hline 382 & $\begin{array}{cccccccccccccccccccccccccc}G & K & F & Y & A & S & K & T & F & Y & D & P & T & K & Q & R & R & I & L & W & G & W & I & G & E & T\end{array}$ \\
\hline 4 & ACAATGAAGGTGATGATCTTTIGAAGGGTTGGGCATGTGITCAGGTATCACTCATCTATCATCGTACCATAATGAGCA \\
\hline 08 & $\begin{array}{llllllllllllllllllllllllllll}D & N & E & G & D & D & L & L & K & G & W & A & C & V & Q & V & S & L & I & Y & H & R & I & I & M & S\end{array}$ \\
\hline & [TGATAATATCCCATAGAAAAAGATCGAAACTAAATATATGTACACTACGATGTGACTGTTTATAGGCAATGTTCTA \\
\hline 34 & * \\
\hline 405 & ГСТААТ \\
\hline 83 & AAA \\
\hline
\end{tabular}

Fig. 2. Nucleotide and deduced amino acid sequence of full-length RhSAIl cDNA from Rhododendron. Deduced amino acid sequences are under the nucleotide sequence with a one-letter code, The asterisk means the stop codon, and the highlight is gene-specific primers sequence for detection by real-time quantitative polymerase chain reaction. This cDNA GenBank accession number is JX261974. 


\begin{tabular}{|c|c|}
\hline & ATGAACTCCCATTCTTCCCCTCCTGAAGACCTGGAAAGATCGGCO \\
\hline & 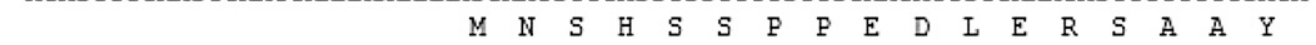 \\
\hline & ICGGCTGCCGGACCACCCTCCCTCTCCGGCGGATCACCGCCGACCCTTCAAGGGTCTCGTCGGAATATTCGTCT \\
\hline & 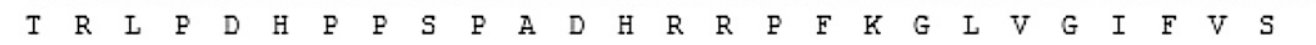 \\
\hline 57 & TGCTCTTAATGTCGTCTTTGGTTGCTTTAATCCTCAATCAAGACCCTCGTCCCCGGTCTAACGTCAATGACGACCAA \\
\hline & 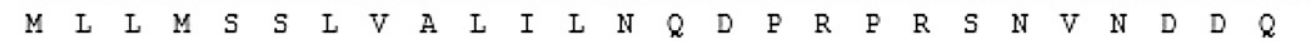 \\
\hline & GGAGAGTACTTCTCCTICAATGCCGGTGCCGGATAGTTTAATGCCGCCGICGAGGGGGGTGGCGCAGGGGGTGIC \\
\hline & $\begin{array}{llllllllllllllllllllllllll}R & E & S & T & S & P & S & M & P & V & P & D & S & L & M & P & P & S & R & G & V & A & Q & G & S\end{array}$ \\
\hline & AGAAGGCGGTCCGGGAATTCTCCGGTAGCGGCCCGGTTTICCCTTGGACTAATGCTATGCTGGCTIG̈GCAGAGGACI \\
\hline & 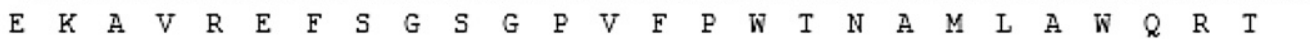 \\
\hline & CTTACCATTTTCAGCCGGAAAAAAACTGGATGAATGATCCTGATGGTCCATTGCATCACATGGGATGGTACCACCTTT \\
\hline & 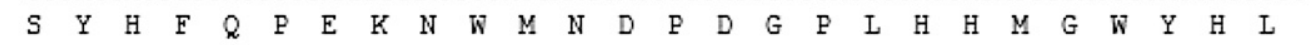 \\
\hline & TACCAGTACAACCCCGATTCAGCAATTTGGGGCAACATAACATGGGGCCACGCAGTATCAAGGGACCTGATCCACT \\
\hline & $\begin{array}{llllllllllllllllllllllllllll}F & Y & Q & Y & N & P & D & S & A & I & W & G & N & I & T & W & G & H & A & V & S & R & D & L & I & H\end{array}$ \\
\hline & GGCTCTACCTCCCCATCGCCATGGTCCCCGATCACTGGTTCGATTTGAACGGTGTTTGGACTGGGTCTGCTACCCTO \\
\hline & 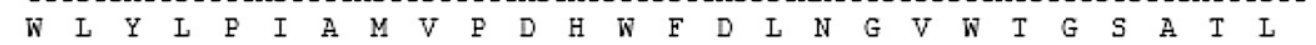 \\
\hline & TCCCCGACGGTCAGATCATCATGTIGTACACCGGTGATACCGATAACGCCGTGCAGGTGCAAAACCTAGCGTATCCCG \\
\hline 10 & 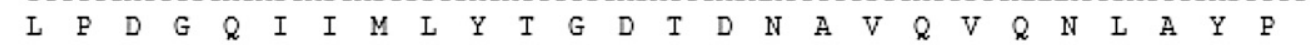 \\
\hline 93 & CCAACTTATCTGATCCCCTCCTCCTAGATTGGGTCAAGTATGAACAAAACCCGGTAATTGTTCCCCCACCCGGAATTG \\
\hline & $\begin{array}{llllllllllllllllllllllll}\text { A } & N & \text { L } & \text { S } & \text { D } & \text { P } & \text { L } & \text { L } & \text { L } & \text { D } & \text { W } & \text { V } & \text { K } & \text { Y } & \text { E } & Q & \text { N } & \text { P } & \text { V } & \text { I } & \text { V } & \text { P } & \text { P } & \text { P }\end{array}$ \\
\hline 81 & GACTAACGTATTITCGGGACCCGAGTACAGCATGGTACGCCCAAGAAGGCACATGGCGGGTCGCTATCGGGTCAAAGG \\
\hline & $\begin{array}{llllllllllllllllllllllllllll}G & L & T & Y & F & R & D & P & S & T & A & W & Y & A & Q & E & G & T & W & R & V & A & I & G & S & K\end{array}$ \\
\hline & AATAAAACGGGTATTGCCCTTGTGTACCAAACCACTAATTTTACTAGCTTCGGGCTTATGGATGGGGTAATGCATG \\
\hline 18 & 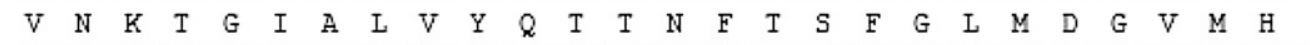 \\
\hline & CGGTTCCGGGTACAGGTATGTGGGAGTGCATAGATATAACCCGGAGAACGACAAGTGACGCAAATGGGCTGGACACGT \\
\hline 04 & 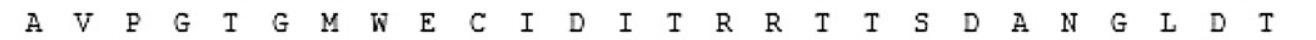 \\
\hline & CGTTCAATGGGCCGGGTATTAAGCATGTCCTGAAGGCAAGCTTAGATAATGAGAAGAAGGATTATTATGCAATTGGGA \\
\hline 30 & 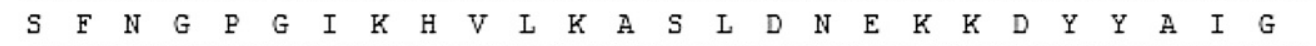 \\
\hline & CATATGATCCTGTAAACAACACGTGGACGCCTGACAACCCGGAAATGGACGTGGGTATCGGGTTGCGGGTGGATTATG \\
\hline & $\begin{array}{llllllllllllllllllllllllllll} & \mathrm{Y} & \mathrm{D} & \mathrm{P} & \mathrm{V} & \mathrm{N} & \mathrm{N} & \mathrm{T} & \mathrm{W} & \mathrm{T} & \mathrm{P} & \mathrm{D} & \mathrm{N} & \mathrm{P} & \mathrm{E} & \mathrm{M} & \mathrm{D} & \mathrm{V} & \mathrm{G} & \mathrm{I} & \mathrm{G} & \mathrm{L} & \mathrm{R} & \mathrm{V} & \mathrm{D} & \mathrm{Y}\end{array}$ \\
\hline & GAGTGTACTATGCATCAAAGACGTTTTATGACCAGAACAAGCAGAGGAGGATCTCGTGGAGTTGGATCGGAGAAACTC \\
\hline & 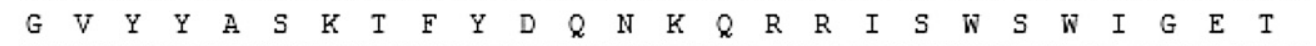 \\
\hline & ATAATGAAAGTGATGACCTITTGAAGGGTTGGGCATCAGTTCAGACCATTCCGAGGACGGTAGTGTTTGACAAGAAGA \\
\hline 08 & 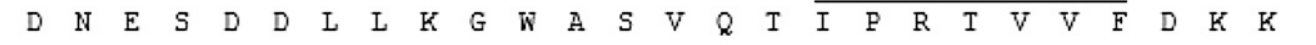 \\
\hline & CCGGAAGCAACATACTTCAATGGCCAGCGGAAGAAGTGGAGAGGTTGCGATTGAACGTTACTGAATICAATGGAGTGG \\
\hline & 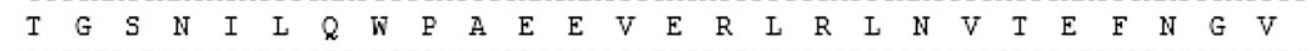 \\
\hline & AGCTTGGTCCGGGATCAGTTGTGCCACTGAACATAAGCTCGGCTACACAGTTGGACATAGTCGCTACATTTGAAGTTG \\
\hline 60 & $\begin{array}{lllllllllllllllllllllllllll}E & L & G & P & G & S & V & V & P & I & N & I & S & S & A & I & Q & L & D & I & V & A & I & F & E\end{array}$ \\
\hline & AAGGCGGCGCTGGAGGCGACAACTGAAGCCGATGCTGGCCACACATGCAGCACCACCGGTGGTGCTGTATCAAGA \\
\hline 186 & 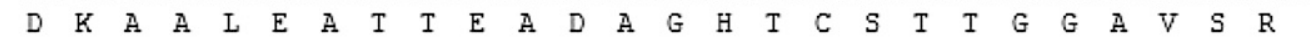 \\
\hline & GCGCTITGGGACCTITCGGGCTGCTGGTTCTCGCTGATGAATCGTTATCCGAGTTAACGCCTGICTATTTCTACATIT \\
\hline & $\begin{array}{llllllllllllllllllllllllll}G & A & L & G & P & F & G & L & L & V & L & A & D & E & S & L & S & E & L & T & P & V & Y & F & Y & I\end{array}$ \\
\hline & AAGTTCATTGACGGCAGTTACAAGACCTTCTTCTGTTCCGATGAGATGAGGTCATCAAAGGCTTCATCAGTTAAC \\
\hline 53 & $\begin{array}{lllllllllllllllllllllllllll}S & K & F & I & D & G & S & Y & K & T & F & F & C & S & D & E & M & R & S & S & K & A & S & S & V & N\end{array}$ \\
\hline & AGAGGGTGAATGGTGGCACGGTCCCTGTGCTCGAGGGCGAAAAATACTCTATGCGGTTATTGGTGGATCATTCGATI \\
\hline 56 & 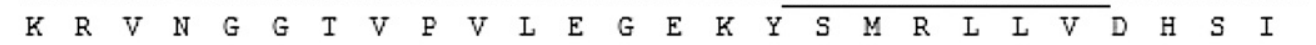 \\
\hline & TGGAGAGCTTIGCTCAAGGAGGAAGAACAGTGATCACGTCTCGAATTTACCCAACGAGGGCCATCGATGGAGCAGCGC \\
\hline & 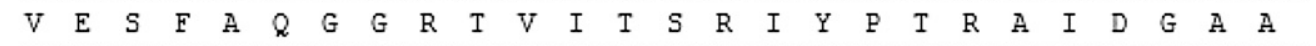 \\
\hline & GAGTATTTCTGTTCAACAATGCCACCGGAACAAATGTAACAGCATCTCTGAAGATATGGCAAATGGATTCAGCACAC \\
\hline 616 & 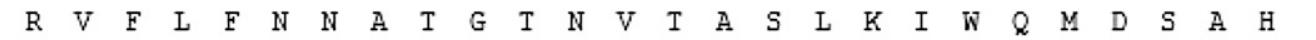 \\
\hline & TTCACTAATTCTTGTTGGATCAGATGIAGTTCACTCTGGTCAATTCATCCCTTTTCCTCGTTTGGTTGTCTGGAAAAI \\
\hline & * \\
\hline & AAAAATCTGGAATTTCGCAGCAACCAAACTCCGATATTGTGTGTAAAATCCCTTT \\
\hline & ITGTCTAAAGGCATTATATAATAATTAGTTGTAGATCATTTGAACAGTGAGTATTGGAAT \\
\hline & \\
\hline
\end{tabular}

Fig. 3. Nucleotide and deduced amino acid sequence of full-length RhSAI2 cDNA from Rhododendron. Deduced amino acid sequences are under the nucleotide sequence with a one-letter code. The asterisk means the stop codon, and the highlight is gene-specific primers sequence for detection by real-time quantitative polymerase chain reaction. This cDNA GenBank accession number is JX261973.

SAI activity was determined by monitoring the quantification of reducing sugars generated at $510 \mathrm{~nm}$ following the method of Sood et al. (2006).

Statistical analysis. All data were means of three replicates at least with SDS. The results were analyzed for variance using the SAS/STAT statistical analysis package (Version 6.12; SAS Institute, Cary, NC).

\section{Results}

Cloning a CDNA ENCoding SAI from Rhododendron. The cDNA encoding RhSAIl was $1511 \mathrm{bp}$ in length and contained an open reading frame of $1329 \mathrm{bp}$, encoding a protein of 422 amino acids (Fig. 2). The cDNA encoding RhSAI2 was $2230 \mathrm{bp}$ in length and contained an open reading frame of $1956 \mathrm{bp}$, 

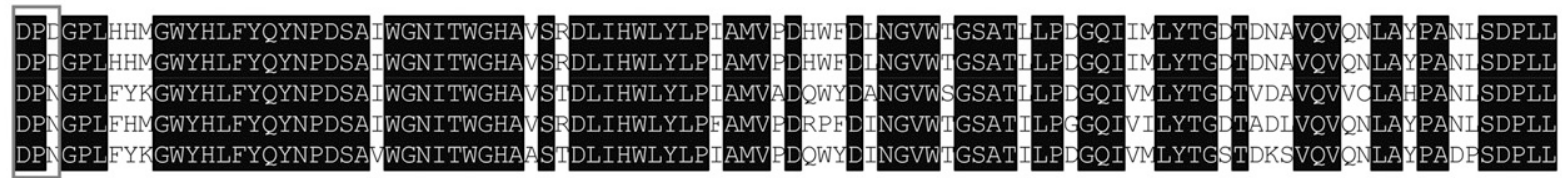

RHSAI 1

RHSAI2

RHSAI

CCSAI

CSSAI
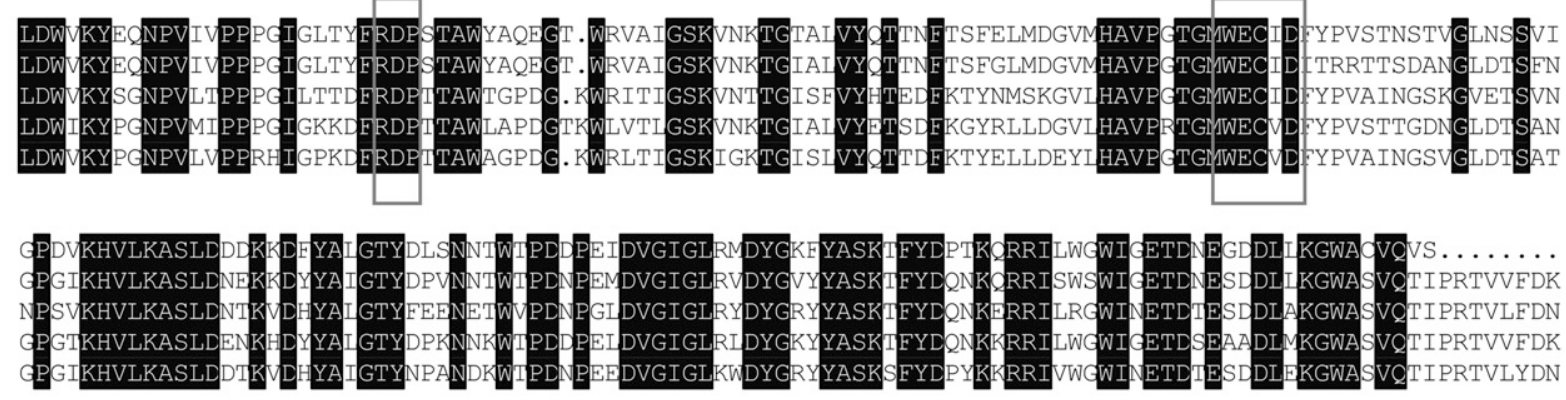

Fig. 4. Alignment of deduced protein sequences of soluble acid invertase (SAI) genes from different plants. The GenBank accession numbers for the SAI sequences are: RhSAII (JX261974) from Rhododendron hybrid 'Yuqilin', RhSAI2 (JX261973) from Rhododendron 'Yuqilin', RhSAI (JN592032) from Rosa hybrida, CcSAI (DQ834315) from Coffea canephora, and CsSAI (AB276108) from Citrus siensis. This alignment was produced using the DNAMAN 5.0 program (Lynnon Corp., Vaudreuil, Quebec, Canada). The $\beta$-fructosidase motif N-D-P-(D/N), the putative active site W-E-C-(I/V)-D, and R-D-P are boxed.

encoding a protein of 651 amino acids (Fig. 3). The two SAIs shared $94 \%$ and $89 \%$ identity in nucleotide sequence and amino acid sequence, respectively. A BLAST comparison of their nucleotide sequences with those of invertases showed that the two obtained sequences were similar to vacuolar invertases found in other plants. The deduced amino acid sequences of RhSAII and RhSAI2 both contain highly conserved motifs present in all SAI (Chen et al., 2009), including the $\beta$-fructosidase motif N-D-P-(D/N) the putative active site W-E-C-(I/V)-D, and R-D-P (these motifs are indicated in Fig. 4). The Rhododendron sequences had high similarity $(70 \%$ to $85 \%)$ to nucleotide sequences encoding SAIs derived from Coffea canephora (DQ834315), Citrus siensis (AB276108), and Rosa hybrida (JN592032). An unrooted phylogenetic tree created using the neighbor-joining method in MEGA5.05 was generated using amino acid sequences encoding SAIs and cell-wall invertases (CWIs) from Rhododendron and other plants (Fig. 5) Plant invertase sequences, regardless of their origins from either monocots or dicots, were distinctly separated into soluble acid invertases and CWIs. Both RhSAII and RhSAI2 invertases formed a clade with the other SAI. This analysis suggests that these two invertase genes share a closer evolutionary relationship to SAI from other species than to CWI genes in other species. This also supports the idea that divergence occurring between SAI and CWI enzymes preceded the diversification of broad taxonomic groups during evolution (Kim et al., 2000).

EXPRESSION OF RHSAI1 AND RHSAI2 GENES IN RHODODENDRoN TISSUES. RhSAII and RhSAI2 expression was examined in a cultivar of tissues using Q-PCR analysis (Table 3). For these experiments, expression of PlActin was monitored as an internal control. The expression of RhSAIl transcript in flower petals at Stage I of floral development was used as the comparator sample (1.00) in our Q-PCR analyses. The relative expression ratio of $R h S A I I$ transcript showed its maximum level in stems at 0.5301 and the lowest level in roots at 0.0998 . The expression level of RhSAI2 was significantly lower than 
that of RhSAI1 in all tissues. RhSAI2 was most abundantly expressed in stems followed by petals, roots, and leaves.

EXPRESSION OF RHSAI1 AND RHSAI2 DURING DIFFERENT STAGES OF FLORAL DEVELOPMENT IN RHODODENDRON. To determine the pattern of $R h S A I 1$ and $R h S A I 2$ expression in petals from different stages of floral development, Q-PCR analysis was performed. The result showed that the expressions of $R h S A I l$ and $R h S A I 2$ were significant different during floral development (Fig. 6). RhSAIl was most abundantly expressed at Stage I of floral development, expression decreased to its minimum level at Stage III, and modestly increased at Stage IV. The expression of $R h S A I 2$ presented a different pattern from that of $R h S A I 1$ during floral development. $R h S A I 2$ expression increased gradually during floral development, from a relative value of 0.01 at Stage I until its maximum level of 0.21 at Stage IV.

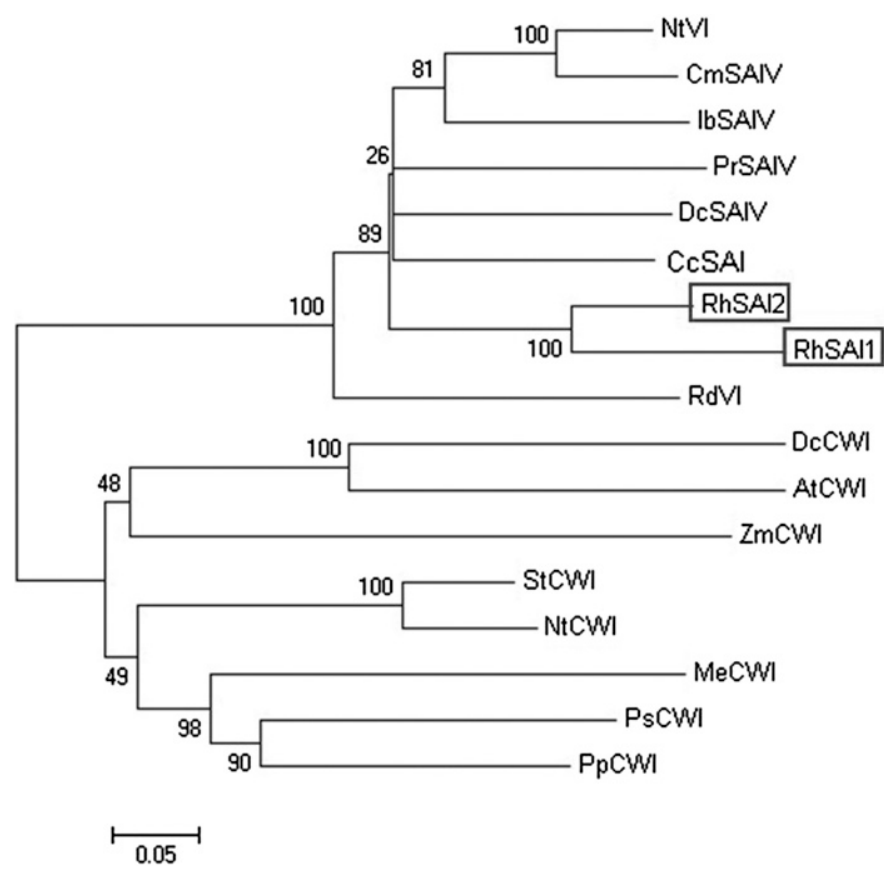

Fig. 5. Phylogenetic tree of RhSAIl and RhSAI2. RhSAII and RhSAI2 cloned in this study are boxed. The dendrogram was built by the MEGA 5.05 program (Tamura et al., 2011) by parsimony with the neighbor-joining method. Sequences used are deduced amino acid sequences from the nucleotide sequences AtCWI (JN790063) from Agave tequilana, CcSAI (DQ834315) from Coffea canephora, CmSAIV (EU260044) from Cucumis melo, DcSAIV, DcCWI (X75351, X78424) from Daucus carota, IbSAIV (AY037938) from Ipomoea batatas, MeCWI (JQ339929) from Manihot esculenta, NtVI, NtCWI (AJ305044, X81834) from Nicotiana tabacum, PpCWI (JQ412748) from Prunus persica, PrSAIV (GU997130) from Phelipanche ramose, PsCWI (X85327) from Pisum sativum, RhSAIl (JX261974) and RhSAI2 (JX261973) from Rhododendron, RdVI (HQ600584) from Rumex dentatus, StCWI (Z22645) from Solanum tuberosum, and ZmCWI (NM_001111899) from Zea mays.
QUANTIFICATION OF SOLUBLE SUGARS AND ACID INVERTASE ACTIVITY. To explore the function of RhSAI in regulating floral development, we measured the soluble sugar content and SAI activity in flower petals from Stage I to Stage IV of development. Sucrose, glucose, and fructose were most abundant at Stage III but their levels decreased at Stage IV (Fig. 7A). In comparison with the other two sugars, the sucrose content of flower petals at Stage IV was reduced most significantly. In contrast to the sugar contents, SAI activity was most abundant during the initial stage (Stage I) and showed a significant decrease by Stage III (Fig. 7B).

\section{Discussion}

Soluble acid invertase, catalyzing the hydrolysis of sucrose into hexose monomers, is thought to play a key role in regulating sugar content to contribute to all aspects of plant growth and development (Jain et al., 2008; Roitsch and Gonzalez, 2004). Early studies suggested that invertases probably contributed to petal senescence by altering the sink strength of sugars in flowers, because its activity was particularly high in young flower petal tissue (Kubo et al., 2001; Sood et al., 2006; Woodson and Wang, 1987). In this article, we cloned and characterized the expression of two cDNAs encoding SAI isoforms ( $R h S A I 1$ and $R h S A I 2)$ from Rhododendron. Our results indicated that SAI was encoded by more than one locus in Rhododendron, which was consistent with reports on other plants (Coupe et al., 2003; Yamada et al., 2007).

Invertase gene expression and function during the processes of plant growth and development such as fruit ripening and

\section{$\square R h S A I 1 \quad \square R h S A I 2$}

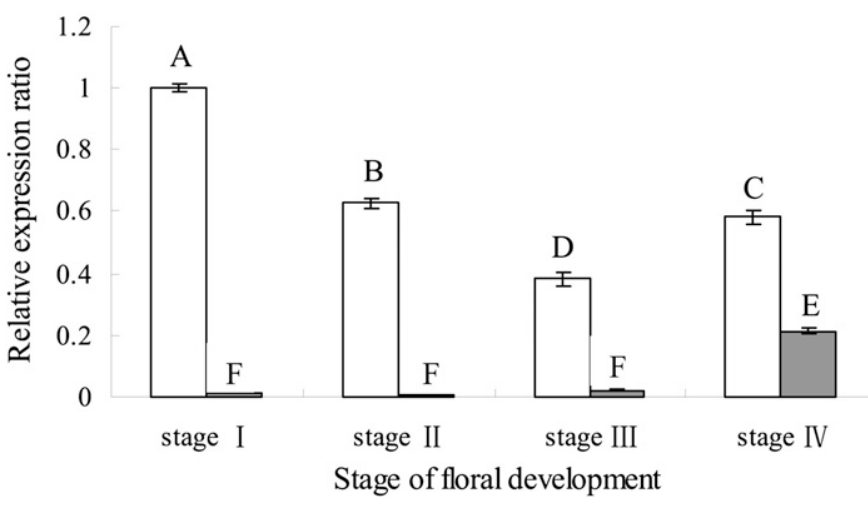

Fig. 6. Relative expression ratio of RhSAII and RhSAI2 gene in petals from different stages of floral development in Rhododendron by real-time quantitative polymerase chain reaction analysis. Bars indicate SE, and different letters indicate significant differences $(P<0.01)$.

Table 3. Relative expression ratio of RhSAI1 and RhSAI2 gene in different tissue at the Stage III of floral development in Rhododendron by realtime quantitative polymerase chain reaction analysis. ${ }^{\mathrm{z}}$

\begin{tabular}{|c|c|c|c|c|}
\hline \multirow[b]{2}{*}{ Gene } & Roots & Stems & Leafs & Petals \\
\hline & \multicolumn{4}{|c|}{ Relative expression ratio $(\text { mean } \pm \mathrm{SE})^{\mathrm{y}}$} \\
\hline RhSAII & $0.0998 \pm 0.0021 \mathrm{~d}$ & $0.5301 \pm 0.0260 \mathrm{a}$ & $0.1407 \pm 0.0097 \mathrm{c}$ & $0.3831 \pm 0.0236 \mathrm{~b}$ \\
\hline RhSAI2 & $0.0013 \pm 0.0000 \mathrm{e}$ & $0.0252 \pm 0.0002 \mathrm{e}$ & $0.0009 \pm 0.0002 \mathrm{e}$ & $0.0207 \pm 0.0020 \mathrm{e}$ \\
\hline
\end{tabular}

${ }^{\mathrm{z}}$ The expression of RhSAII transcript in flower petals at Stage I of floral development was used as the comparator sample (1.00) for relative gene expression analysis, and all the numbers in the table were proportions of that.

${ }^{y}$ Values within a column followed by different letters indicate significant differences by Duncan's multiple range test at $P<0.01$. 

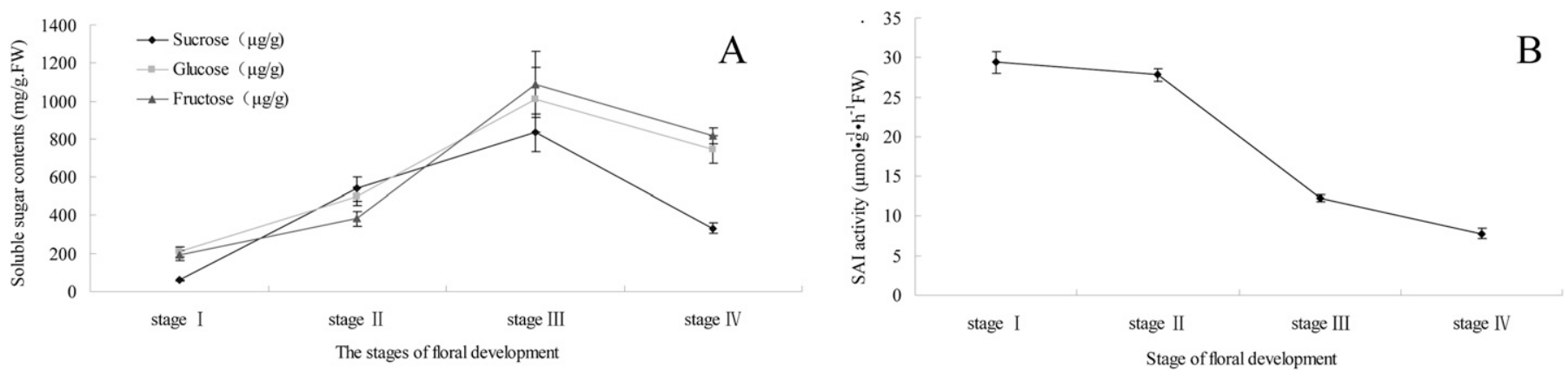

Fig. 7. Soluble sugar contents (A) and soluble acid invertase (SAI) activity (B) in different stages of floral development in Rhododendron. The soluble sugar contents determination and SAI activity assays were carried out on duplicated samples and the experiments were repeated three times. Bars indicate SE.

floral development has become an increasingly important topic of investigation. An invertase gene (MaCwINV1/pBANUU103t) was most abundantly expressed during the middle stages of the ripening of banana (Musa sapientum) fruit, and its expression decreased during the late ripening stage in cultivars IDN 110 and Sowmuk (Fils-Lycaon et al., 2011). Two SAI genes had been cloned from japanese pear (PsS-AIV1 and PsS-AIV2) and expression of both transcripts was high at the young stage before the start of active fruit enlargement and then decreased significantly during fruit maturation (Yamada et al., 2007). We observed unique patterns of SAI expression in Rhododendron. The expression of RhSAII was highest at the earliest stage of floral development (Stage I), subsequently decreased to its lowest at the full-blooming stage (Stage III), and increased somewhat at the end of flowering (Stage IV). In contrast to the $R h S A I 1$ gene, the expression of $R h S A I 2$ gene showed a distinct pattern during floral development. Expression of this gene was at its lowest during Stage I and it subsequently increased until Stage IV, which suggests that the two isoenzymes might have different functions in carbohydrate metabolism during flower development in Rhododendron. This situation also existed in japanese pear (Yamada et al., 2007).

The expression of two $S A I$ isoforms ( $S I$ and $s I I)$ showed spatial differences in carrot [Daucus carota (Sturm et al., 1995)]. The $s I$ was most abundantly expressed in roots and leaves of young plants, whereas $S I I$ showed the highest expression at the middle stage of taproot enlargement. Tian et al. (2009) reported that $\mathrm{CmS}$-AIVI transcripts in muskmelon (Cucumis melo) were abundant in the flowers and fruit but could not be detected in roots, stems, and leaves by Northern blot hybridization analysis. In this study, we demonstrated that two SAI isoforms of Rhododendron were expressed in all organs, showing their highest expression in stem tissue.

Genes encoding SAI enzymes have recently been cloned and characterized from a number of plant species. The relationship between sugar content and SAI activity has been reported in fruit such as sugarcane [Saccharum sinensis (Verma et al., 2011)], japanese pear (Yamada et al., 2007), banana (Fils-Lycaon et al., 2011), and in other plants such as barley [Hordeum vulgare (Nagaraj et al., 2005)] and carrot (Tang et al., 1999). However, the relationship between sugar content and SAI activity in relation to flowers is poorly understood. Sood et al. (2006) reported that invertase activity increased significantly during full bloom, coinciding with high reducing sugar content during this period in roses (Rosa rugosa). In our current study, we measured the soluble sugar content and SAI activity during the entirety of floral development, including the terminal stage (Stage IV).
Soluble sugars began to accumulate significantly during floral development at Stage I and reached their maximum levels at Stage III and thereafter decreased until the end of flowering. The pattern of RhSAIl expression during floral development was basically consistent with the observed changes in SAI activity but was in contrast to the alteration of soluble sugar contents during floral development. The abundant expression of $S A I$ genes and high enzyme activity in young floral tissues might play an important role in supplying substrate energy needed for cell division and growth by hydrolyzing sucrose into hexose monomers (Yamada et al., 2007).

\section{Literature Cited}

Anne, L.R., E.C. Rosanne, M.P. Jai, A.J. Mark, and P.L.G. Christopher. 2011. A soluble acid invertase is directed to the vacuole by a signal anchor mechanism. J. Plant Physiol. 168:983-989.

Chen, T.H., Y.C. Huang, C.S. Yang, C.C. Yang, and A.Y. Wang. 2009. Insights into the catalytic properties of bamboo vacuolar invertase through mutational analysis of active site residues. Phytochemistry 70:25-31.

Coupe, S.A., B.K. Sinclair, L.A. Greer, N.E. Gapper, L.M. Watson, and P.L. Hurst. 2003. Analysis of acid invertase gene expression during the senescence of broccoli florets. Postharvest Biol. Technol. 28:27-37.

Davies, C. and S.P. Robinson. 1996. Sugar accumulation in grape berries. Cloning of two putative vacuolar invertase cDNAs and their expression in grapevine tissues. Plant Physiol. 111:275-283.

Fils-Lycaon, B., P. Julianus, M. Chillet, C. Galas, O. Hubert, D. Rinaldo, and D. Mbéguié-A-Mbéguié. 2011. Acid invertase as a serious candidate to control the balance sucrose versus (glucose + fructose) of banana fruit during ripening. Sci. Hort. 129:197-206.

Hurst, P.L., V. Cheer, B.K. Sinclair, and D.E. Irving. 1997. Biochemical responses of asparagus to controlled atmosphere storage at $20^{\circ} \mathrm{C}$. J. Food Biochem. 20:463-472.

Jain, M., P.S. Chourey, Q.B. Li, and D.R. Pring. 2008. Expression of cell wall invertase and several other genes of sugar metabolism in relation to seed development in sorghum (Sorghum bicolour). J. Plant Physiol. 165:331-344.

Kim, J.Y., A. Mahé, S. Guy, J. Brangeon, O. Roche, P.S. Chourey, and J.L. Prioul. 2000. Characterization of two members of the maize gene family, Incw3 and Incw4, encoding cell-well invertases. Gene 245: 89-102.

Koch, K.E. 1996. Carbohydrate-modulated gene expression in plants. Annu. Rev. Plant Physiol. Plant Mol. Biol. 47:509-540.

Kubo, T., I. Hohjo, and S. Hiratsuka. 2001. Sucrose accumulation and its related enzyme activities in the juice sacs of Satsuma mandarin fruit from trees of different crop loads. Sci. Hort. 91:215-225.

Laia, A. and M.B. Sergi. 2012. Sucrose accelerates flower opening and delays senescence through a hormonal effect in cut lily flower. Plant Sci. 188:41-47. 
Meng, L., L. Zhou, M.Z. Zhang, and S.L. Dai. 2006. An efficient and economic method for preparation total RNA of petals. Biotechnology 16:38-40[in Chinese with English abstract].

Munneé-Bosch, S. and P. Lalueza. 2007. Age-related changes in oxidative stress markers and abscisic acid levels in a drought-tolerant shrub, Cistus clusii grown under Mediterranean field conditions. Planta 225:1039-1049.

Nagaraj, V.J., V. Galati, M. Lüscher, T. Bollerand, and A. Wiemken. 2005. Cloning and functional characterization of a cDNA encoding barley soluble acid invertase (HvINV1). Plant Sci. 168:249258.

Roitsch, T. and M.C. Gonzalez. 2004. Function and regulation of plant invertases: Sweet sensations. Trends Plant Sci. 9:606-613.

Rolland, F., E.B. Gonzalez, and J. Sheen. 2006. Sugar sensing and signaling in plants: Conserved and novel mechanisms. Annu. Rev. Plant Biol. 57:675-709.

Salerno, G.L. and L. Curatti. 2003. Origin of sucrose metabolism in higher plants: When, how and why? Trends Plant Sci. 8:63-69.

Schmittgen, T.D. and K.J. Livak. 2008. Analyzing real-time PCR data by the comparative CT method. Nat. Protoc. 36:1101-1108.

Singh, K.K., L.K. Rai, and B. Gurung. 2009. Conservation of Rhododendron in Sikkim Himalaya: An overview. World J. Agr. Sci. 5:284-296.

Sood, S., D. Vyas, and P.K. Nagar. 2006. Physiological and biochemical studies during flower development in two rose species. Sci. Hort. 108:390-396.

Sturm, A., V. Sebkove, K. Lorenz, M. Hardegger, S. Lienhard, and C. Unger. 1995. Development and organ-specific expression of the genes for sucrose synthase and three isoenzymes of acid b-fructofuranosidase in carrot. Planta 195:601-610.

Tamura, K., D. Peterson, N. Peterson, G. Stecher, M. Nei, and S. Kumar. 2011. MEGA5: Molecular evolutionary genetics analysis using maximum likelihood, evolutionary distance, and maximum parsimony methods. Mol. Biol. Evol. 28:2731-2739.

Tang, G.-O., M. Lüscher, and A. Sturm. 1999. Antisense repression of vacuolar and cell wall invertase in transgenic carrot alters early plant development and sucrose partitioning. Plant Cell 11:177-189.

Tao, J., X.Y. Xu, J. Yu, C. Cao, and G. Liang. 2010. Cloning and expression of cell wall acid invertase gene fragment from poinsettia (Euphorbia pulcherrima wild.). Afr. J. Biotechnol. 9:443-448.

Tian, H.M., Q.G. Kong, Y.Q. Feng, and X.Y. Yu. 2009. Cloning and characterization of a soluble acid invertase-encoding gene from muskmelon. Mol. Biol. Rpt. 36:611-617.

van Doorn, W.G. 2004. Is petal senescence due to sugar starvation? Plant Physiol. 134:35-42.

Verma, A.K., S.K. Upadhyay, M.K. Srivastava, P.C. Verma, S. Solomon, and S.B. Singh. 2011. Transcript expression and soluble acid invertase activity during sucrose accumulation in sugarcane. Acta Physiol. Plant. 33:1749-1757.

Woodson, W.R. and H. Wang. 1987. Invertases of carnation petals. Partial purification, characterization and changes in actively during petal growth. Physiol. Plant. 71:224-228.

Yamada, K., T. Kojima, N. Bantog, T. Shimoda, H. Mori, K. Shiratake, and S. Yamaki. 2007. Cloning of two isoforms of soluble acid invertase of japanese pear and their expression during fruit development. J. Plant Physiol. 164:746-755. 